Bartol Preprint BA-97-48, hep-ph/9710533 (To appear in Phys. Rev. Lett.)

\title{
TeV and Superheavy Mass-Scale Particles from Supersymmetric Topological Defects, the Extragalactic $\gamma$-ray Background, and the Highest Energy Cosmic Rays
}

\author{
Pijushpani Bhattacharjee ${ }^{1,2}$, Qaisar Shafi ${ }^{3}$, and F. W. Stecker ${ }^{1}$ \\ ${ }^{1}$ Laboratory for High Energy Astrophysics, Code 661, NASA/Goddard Space Flight Center, Greenbelt, MD 20771 , USA. \\ ${ }^{2}$ Indian Institute of Astrophysics, Bangalore - 560 034, INDIA. \\ ${ }^{3}$ Bartol Research Institute, University of Delaware, Newark, Delaware 19716, USA.
}

\begin{abstract}
Cosmic topological defects in a wide class of supersymmetric theories can simultaneously be sources of higgs particles of mass comparable to the supersymmetry breaking scale $\sim \mathrm{TeV}$, as well as superheavy gauge bosons of mass $\sim \eta$, where $\eta(\gg 1 \mathrm{TeV})$ denotes the associated gauge symmetry breaking scale. For cosmic strings with $\eta \sim 10^{14} \mathrm{GeV}$, the higgs decay can account for the extragalactic diffuse $\gamma$-ray background above $\sim 10 \mathrm{GeV}$, while the gauge boson decay may explain the highest energy cosmic ray flux above $\sim 10^{11} \mathrm{GeV}$, provided that particle production is the dominant energy loss mechanism for cosmic strings, as recent simulations suggest. By the same token, cosmic strings with $\eta$ much above $\sim 10^{14} \mathrm{GeV}$ will be ruled out.
\end{abstract}

PACS numbers: 98.80.Cq, 12.60.Jv, 98.70.Sa, 98.70.Vc

In a wide class of supersymmetric (SUSY) unified gauge theories, including some versions of effective theories derived from superstrings, certain phase transitions can occur at a temperature comparable to the "soft" supersymmetry breaking scale $\lesssim 1 \mathrm{TeV}$, even though the associated gauge symmetry breaking scale itself may be much larger [1,2]. This occurs rather generically in SUSY theories as a consequence of existence [3] of 'directions' along which the effective potential $V$ of the relevant scalar field is almost flat, i.e., the curvature $\left|V^{\prime \prime}\right|^{1 / 2}$ of the potential is much smaller than the vacuum expectation value $(\mathrm{VEV}) \eta$ of the scalar field out to field values $\sim \eta$. An "almost flat potential" for a (complex) higgs field $\Phi$ after supersymmetry breaking generally has the form [1,2,, , t.5 $V=V_{0}-m_{s}^{2} \phi^{2}+\sum_{n=1}^{\infty} \lambda_{n} m_{\mathrm{Pl}}^{-2 n} \phi^{2 n+4}$, where $\phi \equiv|\Phi|$ and $m_{\mathrm{Pl}} \equiv(8 \pi G)^{1 / 2} \approx 2.4 \times 10^{18} \mathrm{GeV}$. The $\phi^{2}$ term arises from 'soft' supersymmetry breaking, so the mass scale $m_{s}$ is typically $\lesssim 1 \mathrm{TeV}$. The higher order (nonrenormalizable) terms would arise from 'integrating out' particles of Planck mass scales in a 'higher' theory such as superstring theory. The flatness of the potential is due to absence of the $\lambda \phi^{4}$ term familiar in non-SUSY theories. Depending on the strengths of the couplings $\lambda_{n}$, the minimum of the potential, i.e., the VEV $\eta$, can lie anywhere in the range $\sim 10^{9} \mathrm{GeV}$ to $\sim M_{\mathrm{GUT}} \sim 10^{16} \mathrm{GeV}$, the grand unification (GUT) scale $[6]$. The 'height' of the potential is $V_{0} \sim \eta^{2} m_{s}^{2}$.

For temperatures $T$ in the range $m_{s} \ll T \lesssim V_{0}^{1 / 4}$, finite temperature corrections to the potential can hold the $\Phi$ field at $\phi=0$ until $T$ falls below $m_{s}$, at which a phase transition occurs taking $\phi$ to $\eta$. Note that although the higgs scalars in these theories are 'light' with mass $m_{\phi} \sim\left|V^{\prime \prime}\right|^{1 / 2} \sim m_{s} \lesssim 1 \mathrm{TeV}$, the associated gauge bosons have the 'usual' mass $\sim \eta$, which can, in particular, be $\sim M_{\mathrm{GUT}}$, if the higgs under consideration breaks the GUT symmetry. Some cosmological consequences of theories with almost flat potentials (hereafter simply referred to as "flat SUSY" theories) have been considered in Refs. [2, 1,5$]$.

In this Letter, we point out that cosmic topological defects (TDs) [7] such as magnetic monopoles and cosmic strings associated with phase transitions in flat SUSY theories [2,5] can, through their collapse, annihilation, or other processes, be sources of higgs bosons of mass $\sim \mathrm{TeV}$, as well as of gauge bosons of superheavy mass scale $\sim \eta \gg 1 \mathrm{TeV}$, and that the decay products of both these kinds of particles may be observable in the Universe today.

Production of extremely energetic photons, nucleons and neutrinos through decay of massive "X" particles (typically of GUT-scale mass $\sim 10^{16} \mathrm{GeV}$ ) originating from TDs [8 12], is a subject of much current interest as a possible explanation [13 115] of the highest energy cosmic ray (HECR) events at energies $\gtrsim 10^{11} \mathrm{GeV}$ [16]. These TDs have usually been considered within the context of the standard non-SUSY (and non-flat) quartic GUT symmetry-breaking higgs potential [7], for which the relevant phase transition occurs at a temperature $T \sim \eta \sim 10^{16} \mathrm{GeV}$. In this case, the associated GUT gauge bosons as well as higgs bosons, and consequently 
the $\mathrm{X}$ particles 'constituting' the TDs, all have masses of order the GUT scale VEV $\eta \sim 10^{16} \mathrm{GeV}$. In contrast, the new feature in flat SUSY theories is that the $\mathrm{X}$ particles produced by the same TD processes 812 would now be higgs of mass $\sim \mathrm{TeV}$ as well as superheavy gauge bosons of mass $\sim \eta \gg 1 \mathrm{TeV}$. We show that decay of these TD-produced TeV mass-scale higgs may contribute significantly to the extragalactic diffuse $\gamma$-ray background (EDGRB) [17] above a few GeV (which seems to be difficult to explain otherwise in terms of emissions from astrophysical objects; see below), while the superheavy gauge bosons could be a source of the HECR particles. In particular, we show that cosmic strings in flat SUSY theories with $\eta \sim 10^{14} \mathrm{GeV}$ may simultaneously explain both EDGRB above a few GeV and HECR, if $\mathrm{X}$ particle production (rather than gravitational radiation emission) is the dominant energy loss mechanism for cosmic strings - a possibility recently suggested in Ref. [10]. By the same token, cosmic strings with $\eta$ much larger than $10^{14} \mathrm{GeV}$ (and hence GUT-scale cosmic strings with $\eta \sim 10^{16} \mathrm{GeV}$ ) in flat SUSY theories overproduce both EDGRB and HECR, and are, therefore, ruled out. (In this case, cosmic strings with $\eta$ much larger than $10^{14} \mathrm{GeV}$ in non-SUSY theories are also ruled out because they overproduce HECR).

Note that although the higgs in flat SUSY theories are 'light', they are not expected to be produced in accelerators operating at energies well below the energy scale $\sim \eta$ because their coupling to minimal supersymmetric standard model (MSSM) particles, for example, is expected to be suppressed by a factor $\sim m_{\phi} / \eta \ll 1$. Thus, TDs may indeed be the only source of these higgs in the present day Universe.

The rate of $\mathrm{X}$ particle production per unit volume at a time $t$ in the matter dominated epoch from a system of TDs can be generally written in the form \& 8 $d n_{X} / d t=\left(Q_{0} / m_{X}\right)\left(t / t_{0}\right)^{-4+p}$, where $t_{0}$ denotes the present epoch, and $Q_{0}$ is the rate of total energy released per unit volume in the form of $\mathrm{X}$ particles (higgs plus gauge bosons) in the present epoch. (We use natural units, $\hbar=c=1$, throughout.) On general grounds we expect that the total energy released will be roughly equipartitioned between the higgs $\left(Q_{0, \phi}\right)$ and the gauge boson $\left(Q_{0, V}\right)$ modes, and so we will assume that $Q_{0, \phi} \approx Q_{0, V} \approx(1 / 2) Q_{0}$. The dimensionless parameter $p$ is in general different for different systems of TDs [8]. Here we consider the case $p=1$, which is representative of a large class of TD processes including those involving cosmic strings and magnetic monopoles [9 11]. The case in which the $\mathrm{X}$ particles are of heavy mass scales $\sim O(\eta)$ has been considered earlier 8,9,11, 15. Here we consider the effects of the TeV mass-scale higgs $\mathrm{X}$ particles, which we shall assume to be non-relativistic. These higgs would decay on a time scale [1, 4, 5] $\tau \sim$ $6.6 \xi^{-1}\left(\eta / 10^{16} \mathrm{GeV}\right)^{2}\left(1 \mathrm{TeV} / m_{\phi}\right)^{3}$ sec, where $\xi \lesssim 1$ is a numerical factor [5]. For relevant values of $\eta$ and $m_{\phi}$ this decay is essentially 'instantaneous' on cosmological time scales at late epochs of interest to us.

By far the largest number of particles eventually produced by an X would come through the hadronic jet fragmentation of quarks and gluons resulting from its decay (see, e.g., Ref. [18 for arguments concerning the dominance of the hadronic decay channel) [19]. The fragmentation of the quarks/gluons into jets of hadrons and the photon spectrum resulting from decay of neutral pions in these jets are well described by the string fragmentation scheme implemented in the JETSET program [20]. We assume typical hadronic 3-body decays 18 of a X particle into all kinematically available quark pairs and one uncolored (assumed massless) spectator, and obtain the injected photon spectrum from the decay of a single higgs $\mathrm{X}$ particle by using the parametrization of the photon spectrum derived from JETSET, as described in Ref. [18]. Folding this spectrum with the $\mathrm{X}$ particle production rate then gives us the full injection spectrum.

In our calculation of the predicted total $\gamma$-ray flux today, we have included the effects of electromagnetic cascading and $\gamma-\gamma$ scattering [21, 18], and also included the effect of absorption due to pair production on photons of infrared, optical and ultraviolet backgrounds [22]. The details of these calculations will be given elsewhere. In our numerical calculations we have assumed a spatially flat universe with $\Omega_{0}=1$ and present Hubble constant $H_{0}=75 \mathrm{~km} \mathrm{sec}^{-1} \mathrm{Mpc}^{-1}$.

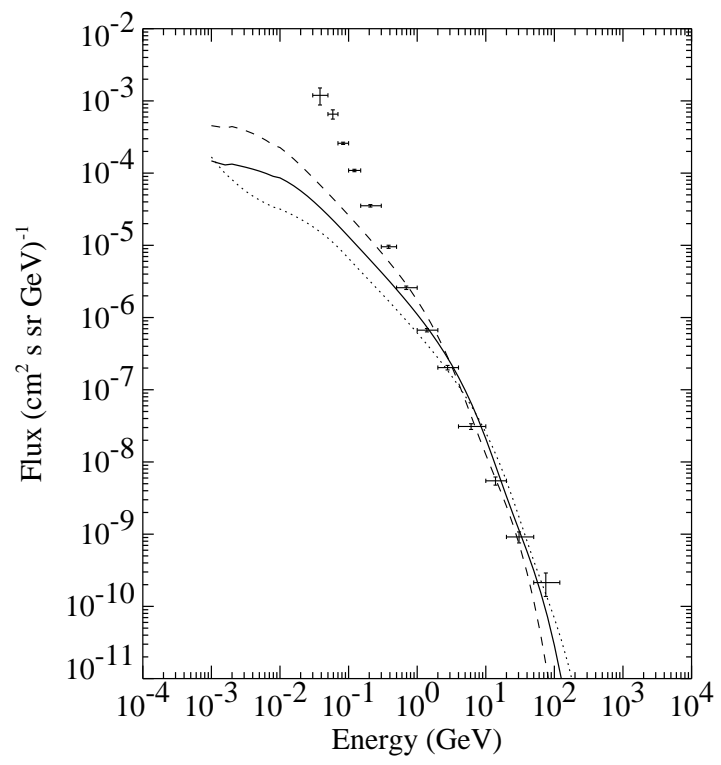

FIG. 1. Gamma ray flux due to decay of the higgs from TD processes with $p=1$, higgs mass $m_{\phi}=500 \mathrm{GeV}$ (dashed curve), $1 \mathrm{TeV}$ (solid curve), and $2 \mathrm{TeV}$ (dotted curve), and $Q_{0, \phi}=4.5 \times 10^{-23} \mathrm{eV} \mathrm{cm}^{-3} \mathrm{sec}^{-1}$. The extragalactic diffuse $\gamma$-ray background data from EGRET (Ref. [17]) are also shown for comparison. 
Fig.1 shows the higgs contribution to the EDGRB for TD processes with $p=1$ and higgs mass $m_{\phi}=500 \mathrm{GeV}$, $1 \mathrm{TeV}$, and $2 \mathrm{TeV}$. The normalization of the curves in Fig. 1 corresponds to $Q_{0, \phi} \simeq 4.5 \times 10^{-23} \mathrm{eV} \mathrm{cm}^{-3} \mathrm{sec}^{-1}$, which, as evident from Fig. 1, is an upper limit on $Q_{0, \phi}$ (and hence on $Q_{0}$ ) for $p=1$ TD processes imposed by the EGRET data.

A recent analysis 17 of the EDGRB indicates that the spectrum continues at least up to $\sim 100 \mathrm{GeV}$. The EDGRB up to $\sim 10 \mathrm{GeV}$ can be interpreted as arising from a superposition of unresolved blazars [23]. However, if blazar $\gamma$-rays are produced by Compton upscattering of lower energy blazar photons, then only X-ray selected BL Lac objects, a small fraction of the blazar population, may qualify as possible contributors to EDGRB above 10 $\mathrm{GeV}$, because only they may have the requisite luminosities. The majority of radio selected BL Lac objects and flat spectrum radio quasars are likely to have luminosities falling off above $\sim 10 \mathrm{GeV}$ [24]. Also, extragalactic absorption effects are likely to steepen the high energy spectra of high-redshift quasars above $\sim 10 \mathrm{GeV}[22]$. Thus, there may be cause to consider another component of cosmic diffuse $\gamma$-ray emission. From the shapes of the curves in Fig.1, we see that decays of higgs of mass $\sim 500-1000$ $\mathrm{GeV}$ from $\mathrm{p}=1 \mathrm{TD}$ processes in flat SUSY theories may play an important role in producing the EDGRB in the $10-100 \mathrm{GeV}$ energy range. The rate of energy injection in the form of TeV higgs needed to explain the EDGRB above a few $\mathrm{GeV}$ is $Q_{0}^{\mathrm{EDGRB}} \simeq 4.5 \times 10^{-23} \mathrm{eV} \mathrm{cm}^{-3} \mathrm{sec}^{-1}$ for $m_{\phi} \simeq 1 \mathrm{TeV}$.

The upper limit on $Q_{0, \phi}$ from EDGRB also implies (through energy equipartition arguments) an upper limit on $Q_{0}$ as well as on $Q_{0, V}$, i.e., $Q_{0} / 2 \approx Q_{0, \phi} \approx Q_{0, V} \lesssim$ $4.5 \times 10^{-23} \mathrm{eV} \mathrm{cm}^{-3} \mathrm{sec}^{-1}$. There is, however, an independent upper limit imposed on $Q_{0, V}$ (and hence on $\left.Q_{0}\right)$ by the HECR data: $Q_{0, V} \lesssim Q_{0}^{\mathrm{HECR}} \simeq 3.3 \times$ $10^{-22}\left(\eta / 10^{16} \mathrm{GeV}\right)^{0.5} \mathrm{eV} \mathrm{cm}^{-3} \mathrm{sec}^{-1}$ [25], where $Q_{0}^{\mathrm{HECR}}$ is the rate of energy injection needed to explain the HECR. For $\eta>1.9 \times 10^{14} \mathrm{GeV}$, we overproduce EDGRB if we wish to explain the HECR, and is, therefore, unfavored. (Of course, TDs with $\eta>1.9 \times 10^{14} \mathrm{GeV}$ can give significant contribution to the EDGRB while not contributing significantly to the HECR.) For $3 \times 10^{11} \mathrm{GeV} \ll$ $\eta<1.9 \times 10^{14} \mathrm{GeV}$ we can produce the HECR, but in this case we significantly underproduce EDGRB if $\eta \ll 1.9 \times 10^{14} \mathrm{GeV}$. The two independent upper limits can be saturated, i.e., we can explain both EDGRB above a few $\mathrm{GeV}$ and HECR, if $\eta \approx 1.9 \times 10^{14} \mathrm{GeV}$.

The value of $Q_{0}$ for a general TD process is not known a priori - it depends on at least two (not necessarily mutually independent) unknown parameters, namely, the fraction of the total energy density of the relevant defects going into $\mathrm{X}$ particles, and the symmetry breaking scale $\eta$ at which the relevant TDs are formed. Therefore, the above arguments do not by themselves rule out the exis- tence of GUT scale (i.e., $\eta \sim 10^{16} \mathrm{GeV}$ ) TDs in general - they only tell us that GUT scale TDs are unlikely to be responsible for HECR, because that would conflict with EDGRB.

The situation is, however, very different in the case of cosmic strings with the recent results 10 of numerical simulations of cosmic string evolution in the Universe. These studies show that the energy density, $\rho_{s}(t)$, in "long" (i.e., horizon crossing) strings at any time $t$ is maintained in the scaling solution [7], $\rho_{s}(t)=\mu /\left(x^{2} t^{2}\right)$, by energy loss from long strings occurring predominantly on the scale of the string width, i.e. through formation of string width-size small loops which quickly decay into $\mathrm{X}$ particles or through direct emission of the $\mathrm{X}$ particles that 'constitute' the strings, and not through formation of (sub)horizon size loops and their subsequent decay by emission of gravitational radiation as thought earlier [7]. (Here $\mu$ is the energy per unit length of the string, and $x$ is in the range $0.27-0.34$ 10].) This result, while subject to confirmation by independent simulations, obviously has important implications for HECR and EDGRB. Indeed, in this case, there is effectively only one free parameter (namely, $\mu$ or equivalently, $\eta$ ) in the problem, which also fixes $Q_{0}$. In fact, in this case, the observed data on ultrahigh energy cosmic rays already rule out [9.10] GUT scale cosmic strings for the standard non-flat potential case. We shall see that this is also true for the flat potential case, but here an additional constraint (due to TeV mass scale higgs) comes from the EDGRB data.

From the results of Ref. [10], the rate of energy loss of strings per unit volume through $\mathrm{X}$ particle emission is $d \rho_{X} / d t \simeq(2 / 3) \mu /\left(x^{2} t^{3}\right)$, which gives $Q_{0} \simeq 7.4 \mu / t_{0}^{3}$, with $x \simeq 0.3$. Requiring $Q_{0, \phi} \approx 0.5 Q_{0} \lesssim Q_{0}^{\mathrm{EDGRB}} \simeq$ $4.5 \times 10^{-23} \mathrm{eV} \mathrm{cm}^{-3} \mathrm{sec}^{-1}$, we get $\mu \lesssim 4.5 \times 10^{-5} \times$ $\left(10^{16} \mathrm{GeV}\right)^{2}$. Taking, for flat potentials, $\mu \sim 0.1 \eta^{2}$ [5], we get $\eta \lesssim 2.1 \times 10^{14} \mathrm{GeV}$. Thus, in this case, GUT scale cosmic strings necessarily overproduce EDGRB, and are, therefore, ruled out. A similar constraint follows from HECR: Here one requires $Q_{0, V} \approx 0.5 Q_{0} \lesssim Q_{0}^{\mathrm{HECR}} \simeq$ $3.3 \times 10^{-22}\left(\eta / 10^{16} \mathrm{GeV}\right)^{0.5} \mathrm{eV} \mathrm{cm}^{-3} \mathrm{sec}^{-1}$, which gives $\eta \lesssim 2.2 \times 10^{14} \mathrm{GeV}$

Note that for a non-flat potential, where $\mu \simeq \pi \eta^{2}$ and where one expects the $\mathrm{X}$ particles to be predominantly of heavy mass scale $\sim \eta$, so that $Q_{0, V} \approx Q_{0}$, the constraint on $\eta$ from HECR is $\eta \lesssim 1.4 \times 10^{13} \mathrm{GeV}$.

It is thus clear that cosmic strings with $\eta$ much greater than $10^{14} \mathrm{GeV}$, and in particular, GUT scale cosmic strings with $\eta \sim 10^{16} \mathrm{GeV}$, are ruled out both for flat as well as non-flat potentials if $\mathrm{X}$ particle production is their dominant energy loss mechanism. At the same time, in this case, cosmic strings with $\eta \sim 2 \times 10^{14} \mathrm{GeV}$ in SUSY models with flat potentials can potentially account for the high energy ends of both EDGRB and HECR. In this respect, absence of free parameters other than the symmetry breaking scale $\eta$ seems to make cosmic strings a "natural" candidate source of HECR (and possibly of 
EDGRB above a few $\mathrm{GeV}$ ).

Cosmic string formation at $\eta \sim 10^{14} \mathrm{GeV}$ rather than at the GUT scale of $\sim 10^{16} \mathrm{GeV}$ is not hard to implement. For example, in a SUSY theory, the breaking $\mathrm{SO}(10) \rightarrow \mathrm{SU}(3) \times \mathrm{SU}(2) \times \mathrm{U}(1)_{\mathrm{Y}} \times \mathrm{U}(1)$ can take place at the GUT unification scale $M_{\mathrm{GUT}} \sim 10^{16} \mathrm{GeV}$ without any cosmic string formation, but the second $\mathrm{U}(1)$ can be subsequently broken with a flat potential with a VEV $\eta \sim 10^{14} \mathrm{GeV}$ to yield cosmic strings that are relevant for EDGRB and HECR.

Cosmic strings with $\eta \sim 10^{14} \mathrm{GeV}$ would be too light to be relevant for structure formation in the Universe. Their signatures on the CMBR sky would also be too weak to detect. However, signatures of these cosmic strings may be searched for with next generation $\gamma$-ray instruments such as GLAST [26, which will be able to resolve further the discrete source component and thereby reveal the possible existence of a truly cosmic component of the EDGRB as in Fig. 1, and in proposed HECR observatories such as Auger 27. and OWL 28].

We wish to thank P. Sreekumar for stimulating discussions on EDGRB, and Graham Kribs, Subir Sarkar and Günter Sigl for useful correspondence. P.B. acknowledges support under a NAS/NRC Senior Research Associateship at NASA/GSFC. Q.S. acknowledges the DOE support under grant DE-FG02-91ER40626.

[1] G. Lazarides, C. Panagiotakopoulos, and Q. Shafi, Phys. Rev. Lett. 56, 557 (1986); Phys. Lett. B 183, 289 (1987).

[2] G. Lazarides, C. Panagiotakopoulos, and Q. Shafi, Phys. Rev. Lett. 58, 1707 (1987).

[3] See for instance, M. Dine et al, Nucl. Phys. B 259, 549 (1985); G. Dvali and Q. Shafi, Phys. Lett. B 339, 241 (1994).

[4] D.H. Lyth and E.D. Stewart, Phys. Rev. Lett. 75, 201 (1995); Phys. Rev. D 53, 1784 (1996).

[5] T. Barreiro, E.J. Copeland, D.H. Lyth, and T. Prokopec, Phys. Rev. D 54, 1379 (1996).

[6] See, e.g., C. Giunti, C.W. Kim, and U.W. Lee, Mod. Phys. Lett. A6, 1745 (1991).

[7] A. Vilenkin and E.P.S. Shellard, Cosmic Strings and other Topological Defects (Cambridge Univ. Press, Cambridge, 1994); M. Hindmarsh and T.W.B. Kibble, Rep. Prog. Phys. 58, 477 (1995).

[8] P. Bhattacharjee, C.T. Hill, and D.N. Schramm, Phys. Rev. Lett. 69, 567 (1992).

[9] P. Bhattacharjee and N.C. Rana, Phys. Lett. B 246, 365 (1990).

[10] G. Vincent, N.D. Antunes, and M. Hindmarsh, Phys. Rev. Lett. (1998) (in press) (hep-ph/9708427); G. R. Vincent, M. Hindmarsh, and M. Sakellariadou, Phys. Rev. D 56, 637 (1997).

[11] C.T. Hill, Nucl. Phys. B 224, 469 (1983); P. Bhattachar- jee and G. Sigl, Phys. Rev. D 51, 4079 (1995); V. Berezinsky and A. Vilenkin, Phys. Rev. Lett. 79, 5202 (1997).

[12] C. T. Hill, D. N. Schramm, and T. P. Walker, Phys. Rev. D 36, 1007 (1987); M. Mohazzab and R. Brandenberger, Int. Jour. Mod. Phys. 2, 183 (1993) and references therein; A. J. Gill and T. W. B. Kibble, Phys. Rev. D 50, 3660 (1994).

[13] G. Sigl, D.N. Schramm, and P. Bhattacharjee, Astropart. Phys. 2, 401 (1994); G. Sigl, S. Lee, D. N. Schramm, and P. Bhattacharjee, Science, 270 (1995); J. W. Elbert and P. Sommers, Astrophys. J. 441, 151 (1995).

[14] G. Sigl, S. Lee, D. N. Schramm, and P. Coppi, Phys. Lett. B 392, 129 (1997) (SLSC); F.A. Aharonian, P. Bhattacharjee, and D.N. Schramm, Phys. Rev. D 46, 4188 (1992).

[15] R.J. Protheroe and T. Stanev, Phys. Rev. Lett. 77, 3708 (1996); 78, 3420 (1997) (E) (PS).

[16] D.J. Bird et al, Phys. Rev. Lett. 71, 3401 (1993); Astrophys. J. 441, 144 (1995); N. Hayashida et al, Phys. Rev. Lett. 73, 3491 (1994); S. Yoshida et al, Astropart. Phys. 3, 105 (1995).

[17] P. Sreekumar et al, Astrophys. J. 494, 523 (1998); P. Sreekumar, F. W. Stecker, and S. C. Kappadath, in Proc. Fourth Compton Symposium, Eds. C. D. Dermer, M. S. Strickman and J. D. Kurfess (AIP Conf. Proc. 410, 1997) (astro-ph/9709258.

[18] G.D. Kribs and I.Z. Rothstein, Phys. Rev. D 55, 4435 (1997).

[19] Some fraction of the X particle decay products could also be LSPs, the lightest SUSY particles; but they are not relevant here for our consideration of the EDGRB. We also neglect other possible non-hadronic decay channels of X. These approximations may lead to uncertainties in our flux estimates by factors of order unity, which we think is not crucial to our main conclusions.

[20] T. Sjöstrand, Comput. Phys. Commun. 82, 74 (1994).

[21] A.A. Zdziarski, Astrophys. J. 335, 786 (1988); R. Svensson and A. A. Zdziarski, ibid 349, 415 (1990); J. Ellis et al, Nucl. Phys. B 373, 399 (1992).

[22] M.H. Salamon and F.W. Stecker, Astrophys. J. 493, 547 (1998).

[23] F.W. Stecker and M.H. Salamon, Astrophys. J. 464, 600 (1996).

[24] F.W. Stecker, O.C. de Jager and M. H. Salamon, Astrophys. J. 473, L75 (1996).

[25] This value corresponds to an injection spectrum $\propto E^{-1.5}$ and corresponds roughly to the most conservative bound obtained by PS [15]. One should keep in mind that the value of $Q_{0}^{\mathrm{HECR}}$ is uncertain due to a variety of uncertain factors including strength of intergalactic magnetic field, the spectrum of the universal radio background, the injection spectrum and so on, all of which affect the theoretically calculated flux. It also depends somewhat on the way one normalizes the theoretically predicted flux with the data; see, e.g., SLSC [14].

[26] E.D. Bloom, Sp. Sci. Rev. 75, 109 (1996).

[27] J.W. Cronin, Nucl. Phys. B (Proc. Suppl.) 28B, 213 (1992).

[28] J.F. Ormes et al, in Proc. 25th ICRC (Durban, 1997), eds.: M. S. Potgieter et al, 5, 273. 